\title{
Airy Beams for Light-sheet Microscopy
}

Jonathan Nylk ${ }^{1,2}$, Zhengyi Yang ${ }^{1}$, Miguel Preciado ${ }^{1}$, Michael Mazilu ${ }^{1}$, Tom Vettenburg ${ }^{3}$, Clara CollLladó $^{2}$, David E. K. Ferrier ${ }^{2}$, Tomáš Čižmár ${ }^{4}$, Frank. J. Gunn-Moore ${ }^{2}$, Kishan Dholakia ${ }^{1}$

1. SUPA, School of Physics and Astronomy, University of St Andrews, St Andrews, UK

2. School of Biology, University of St Andrews, St Andrews, UK

3. Present address: Department of Bioengineering and Aerospace Engineering, Universidad Carlos III de Madrid, Madrid, Spain

4. Present address: School of Engineering, Physics and Mathematics, University of Dundee, Dundee, UK

Light-sheet microscopy (LSM) is a promising technique for live imaging as it facilitates fast, highcontrast imaging of large volumes with minimal phototoxicity [1]. Variations on this imaging method are required to give high-resolution over large fields-of-view and to enable imaging at greater depths into specimens.

A fundamental limitation of LSM with Gaussian beam illumination is the rapid divergence of the beam which prevents a uniformly thin light-sheet, thus impeding high-resolution imaging over a large volume. The use of Bessel beams for light-sheet illumination has grown in popularity because the inherent propagation-invariance of the Bessel beam can be utilized to produce uniformly thin light-sheets over an extended field-of-view [2] however, the extended transverse structure of the Bessel beam lowers the achievable axial resolution and must be combined with additional techniques such as multi-photon excitation, confocal scanning, or structured illumination (see, for example, [3]) to recover highresolution images.

We present a number of innovations using the Airy beam as an alternative propagation-invariant beam for single-photon excitation LSM. The Airy beam also has an extended transverse structure but, rather than detract from image quality, this transverse structure facilitates high-contrast, high-resolution imaging when combined with a simple, one-dimensional deconvolution algorithm [4]. This technique allows for isotropic high-resolution imaging over a ten-fold larger field-of-view compared to a Gaussian light-sheet, and without additional, unnecessary irradiation of the specimen. Figure 1 compares images obtained using Gaussian, Bessel, and Airy light-sheets. Additionally, the broad distribution of energy across the Airy beam lowers peak power and reduces phototoxicity.

Unlike the Bessel light-sheet, which results from an intrinsically two-dimensional pupil modulation and requires the Bessel beam to be scanned to make a light-sheet, an Airy light-sheet can be formed from a one-dimensional cubic phase modulation with a tilted cylindrical lens. This can lead to an inexpensive and accessible Airy light-sheet microscope [5].

An exponentially decreasing signal across the image can result from absorption and scattering of the light-sheet in large biological samples. Our latest approach shows that an attenuation compensating Airy beam can counteract the effects of absorption [6]. We apply this as a novel single-photon excitation approach to deliver a light-sheet deep into specimens without increasing the unnecessary irradiation of other parts of the specimen. The cylindrical pupil function required to produce an Airy light-sheet which compensates for linear attenuation is $\mathrm{P}(\mathrm{u}, 0)=\exp \left(\sigma_{\mathrm{u}} \mathrm{u}\right) * \exp \left(2 \pi \mathrm{i} \alpha \mathrm{u}^{3}\right)$, where $\mathrm{u}$ is the normalised pupil coordinate orthogonal to the light-sheet, $\alpha$ determines the propagation-invariant length of the Airy light- 
sheet, and $\sigma_{\mathrm{u}}$ is the strength of attenuation compensation. Figure 2 compares the image quality obtained in a strongly absorbing medium with and without attenuation compensated Airy light-sheets.

In summary, we have shown the benefits of Airy beams for LSM. Airy beams can enable highresolution imaging over a large field-of-view and their broad transverse structure ensures low phototoxicity. The Airy beam can be easily generated from a tilted cylindrical lens, thus bringing the benefits of Airy beam LSM to a greater number of end users. The use of attenuation compensated Airy beams is also presented as a novel approach to obviate the effects of absorption and scattering in the sample.

\section{References:}

[1] J. Huisken, and D. Y. R. Stainer, Development 136 (2009), p. 1963-1975.

[2] F. O. Fahrbach and A. Rohrbach, Opt. Express 18 (2010), p. 24229-24244.

[3] L. Gao et al, Cell 151(6) (2012), p. 1370-1385.

[4] T. Vettenburg et al, Nat. Methods 11 (2014), p. 541-544.

[5] Z. Yang et al, Biomed. Opt. Express 5(10) (2014), p. 3434-3442.

[6] M. A. Preciado, K. Dholakia, and M. Mazilu, Opt. Lett 39(16) (2014), p. 4950-4953.
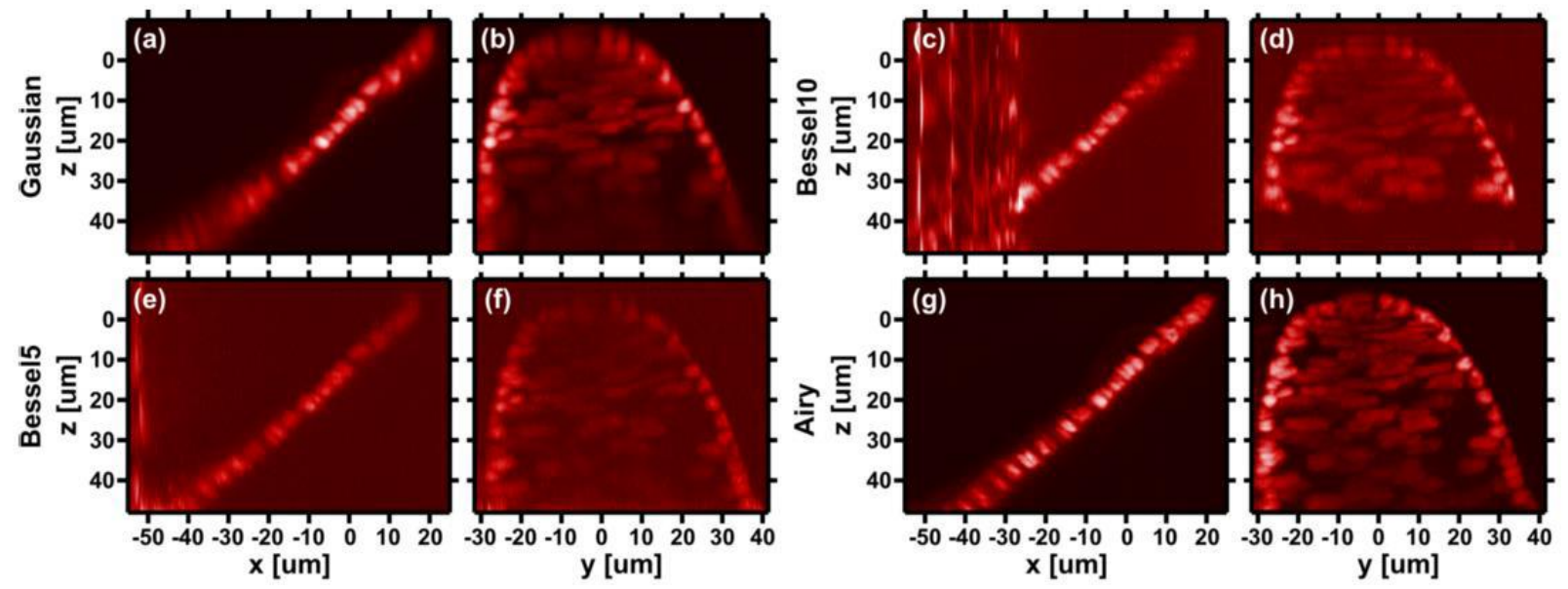

Figure 1. Comparison of various light sheet types imaging the dorsal end of the notochord in a fixed amphioxus (Branchiostoma lanceolatum). Nuclei have been fluorescently labelled with propidium iodide. $y$-axis and $\mathrm{x}$-axis projections, respectively for the case of $(\mathrm{a}, \mathrm{b})$ Gaussian, (c,d) Bessel10, (e,f) Bessel5, and (g,h) Airy beam illumination. Bessel10 and Bessel5 denote Bessel beams with a propagation invariance of \pm 21 and $\pm 42 \mu \mathrm{m}$ from focus of a Gaussian beam respectively.
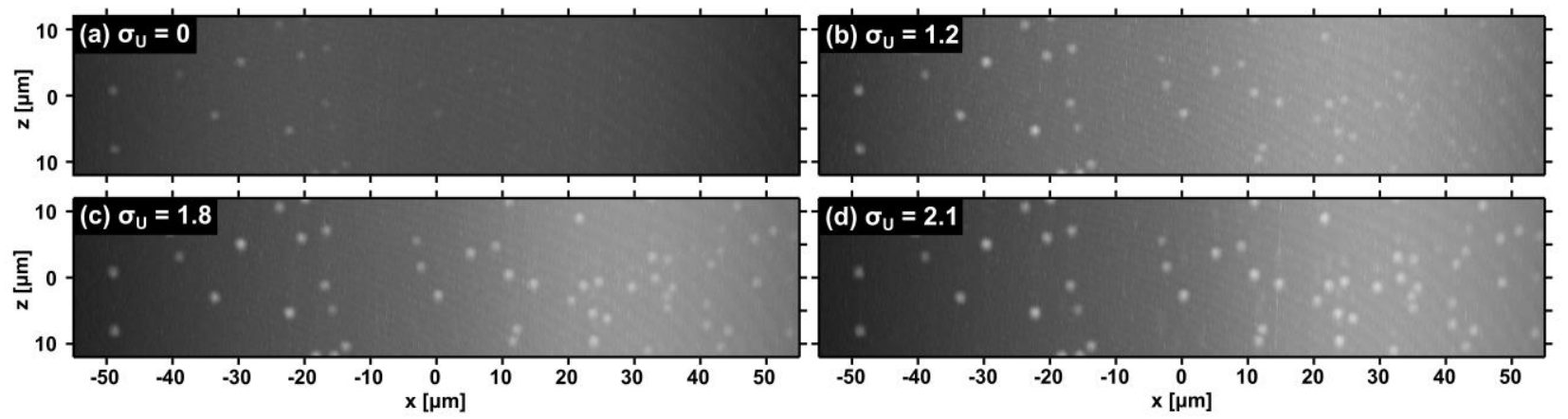

Figure 2. Comparison of attenuation compensated Airy beam light-sheets. $y$-axis projections are shown for fluorescent microspheres embedded in an absorbing medium. 